\title{
La mirada reflexiva, aportaciones epistemológicas al periodismo literario ante el espejismo del metarrelato de la era digital
}

\section{The reflective gaze: epistemological contributions to literary journalism in the face of the meta-narrative illusion of the digital age}
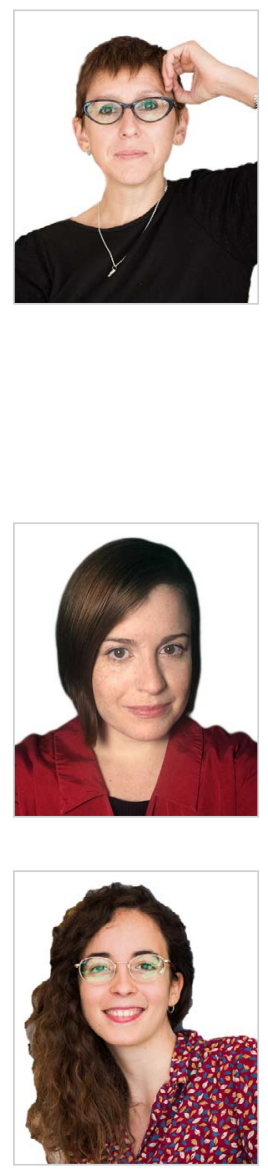

Catalina Gayà Morlà. Doctora en Comunicación y profesora Lectora Serra Hunter del Departamento de Medios, Comunicación y Cultura (UAB). Investigadora de 'Xahrazad. Grupo de pensamiento y acción narrativa en Cultura y Comunicación’ de la UAB. Es profesora de Políticas Culturales en tiempos de capitalismo global en el máster del Departamento, y de Escritura Periodística, en el grado. Hasta 2020, fue consultora de la UOC en el máster de Nuevas Narrativas y profesora de Mirada y Método en el máster de Periodismo Literario de la UAB. Ha dirigido el "Estudio sobre las percepciones en torno a las violaciones machistas y la igualdad entre los jóvenes de 14 a 18 años" para el Instituto Balear de la Mujer; Dona'm la Mar, proyecto del Museo Marítimo de Barcelona en torno a cómo incorporar a las mujeres en sus discursos, y La remor de la memòria, proyecto participativo comunitario del Museo Marítimo de Mallorca. Tiene más de 20 años de experiencia como periodista en diferentes países, entre ellos México, donde trabajó para la revista Cambio, dirigida por Gabriel García Márquez. 'Ve, vivo y cuéntalo' - una enseñanza y un regalo del premio Nobel- ha marcado toda su carrera como periodista e investigadora de discursos no hegemónicos. Es autora, entre otros, de El mar es tu espejo (2017, Libros del K.O) y de Ulises es nombre de mujer. Un relato no oficial del mar (MMB-Diputación de Barcelona, 2021).

Universitat Autònoma de Barcelona, España

catalina.gaya@uab.cat

ORCID: 0000-0001-6190-6824

Cristina Garde. Doctoranda del Departamento de Medios, Comunicación y Cultura (UAB) e investigadora de 'Xahrazad. Grupo de Pensamiento y Acción Narrativa en Cultura y Comunicación' (UAB). Es licenciada en Periodismo por la UAB (2007) y graduada en Artes y Diseño por la Escola Massana (2014). Así mismo, ha cursado el Posgrado en Diseño de Publicaciones Digitales en EINA (2014). Ha trabajado como periodista especializada en sociedad, infografismo y diseño gráfico en El País, El Periódico y Nació Digital y como corresponsal en Bruselas de la Agència Catalana de Notícies (ACN). Ha recibido dos premios Civisme (2018 y 2019) por sendas series de reportajes y ha sido reconocida con el premio Montserrat Roig 2020 por su trayectoria como coordinadora del Social.cat, el diario digital de la acción social en Cataluña. Universitat Autònoma de Barcelona, España

cristina.garde@e-campus.uab.cat

ORCID: 0000-0002-8700-9686

Laia Seró Moreno. Desde el Periodismo y la Etnografía, aborda temáticas sociales con la perspectiva de la literatura documental. Es redactora del diario ARA, doctoranda del Departamento de Medios, Comunicación y Cultura (UAB) e investigadora de 'Xahrazad, Grupo de pensamiento y acción narrativa en Cultura y Comunicación'. Licenciada en Periodismo por la UAB y Máster de Antropología y Etnografía en la Universitat de Barcelona (UB), ha sido miembro del colectivo de reporterismo SomAtents y también ha participado y codirigido investigaciones sociales con perspectiva de género para instituciones como el Museo Marítimo de Barcelona, el Museo Marítimo de Mallorca, el Instituto Balear de la Mujer y el Museo de la Palabra (Mallorca).

Universidad Autónoma de Barcelona, España

Isero@ara.cat

ORCID: 0000-0001-8886-5882

Cómo citar este artículo:

Gayà Morlà, C., Garde, C. y Seró Moreno, L. (2022). La mirada reflexiva, aportaciones epistemológicas al periodismo literario ante el espejismo del metarrelato de la era digital. Doxa Comunicación, 34, pp. 273-289.

https://doi.org/10.31921/doxacom.n34a1477 
Recibido: 21/06/2021 - Aceptado: 10/11/2021 - En edición: 05/12/2021 - Publicado: 01/01/2022

\section{Resumen:}

Este ensayo propone reflexionar en torno a los aportes epistemológicos que la etnografía hace al periodismo literario. El objetivo es incorporar a la discusión sobre el periodismo literario la mirada reflexiva. Se argumenta que un periodismo basado en una mirada reflexiva es un ejercicio participativo en el que los sujetos, mediante un ejercicio de intersubjetividad, intentan interpretar, narrar y crear un mundo común. El ejercicio es dialógico y es fruto de una escucha activa y de la asunción de que la periodista no es ni pasiva ni externa a aquello que vive y narra. La propuesta cambia radicalmente las lógicas de la hipersimulación: permite a la comunidad apropiarse del aquí y ahora y abandonar el presente continuo. En el texto se expone que es función del periodismo literario ser una alternativa al metarrelato del capitalismo acelerado de la era digital y de poner en evidencia el espejismo que construye el capitalismo en las plataformas. La mirada reflexiva ofrece el marco epistemológico para hacerlo.

Palabras clave:

Periodismo literario; mirada; etnografía; reflexividad; mirada reflexiva; metarrelato.
Received: 21/06/2021 - Accepted: 10/11/2021 - Early access: 05/12/2021 - Published: 01/01/2022

\section{Abstract.}

This article reflects on the epistemological contributions of ethnography to literary journalism. The objective is to incorporate the reflective gaze into the debate on literary journalism. It is argued that journalism based on a reflective gaze is a participatory exercise in which subjects, through an exercise of intersubjectivity, try to interpret, narrate and create a world in common. The exercise is dialogical. It is the result of active listening and the assumption that the journalist is neither passive nor external to what she experiences and narrates. The proposal radically changes the logics of hyper-simulation. It allows the community to appropriate the here and now and abandon the continuous present. The article argues that it is the function of literary journalism to be an alternative to the meta-narrative of accelerated capitalism in the digital age and to highlight the illusion constructed on platforms by capitalism. The reflective gaze provides the epistemological framework to do this.

Keywords:

Literary Journalism; gaze; ethnography; reflexivity; reflective gaze; meta-narrative.

\section{El mundo como representación: una introducción a la intersubjetividad}

En el Occidente capitalista -y en toda su área de influencia- el periodismo, entendido como disciplina de construcción simbólica de las sociedades, se encuentra amenazado, apenas visible en el bosque de la comunicación digital. En la era del capitalismo de la vigilancia (Zuboff, 2020), el tiempo lineal de la tradición occidental -Heidegger (1986 [1927]) lo llamará tiempo vulgar-que nos ha posibilitado narrar el mundo ${ }^{1}$ se ha acelerado de tal modo que podemos afirmar que vivimos en un presente continuo (Jameson, 1991) que ha cancelado la temporalidad y que, por lo tanto, no nos permite ni el cambio ni la transformación -ni nada que sea constante- y ni siquiera correlacionarnos.

Además, en este tiempo sin proceso, el capitalismo ha impuesto durante al menos tres décadas una simulación global de apariencia hiperreralista (Baudrillard, 1978), es decir, que nos ha obligado a representarnos el mundo como una suplantación del real por los signos de lo real, lo que Baudrillard define como una "operación de disuasión de todo proceso real por su doble operativo" (1978: 7). En esta simulación global, los mass-media del periodismo industrial, que han adoptado en sus praxis una perspectiva realista ingenua (Bunge, 1985), han sido los grandes aparatos de difusión de esta trampa de dimensiones globales, motivo por el

1 Delimitamos el concepto “mundo" como aquello que podemos narrar, tal como lo entiende Wittgenstein (2009 [1921]: 107): “Los límites de mi lenguaje significan los límites de mi mundo. La lógica llena el mundo; los límites del mundo son también sus límites". Este mundo, por lo tanto, no es tanto el conjunto de los objetos que se pueden percibir mediante la experiencia, y que Kant llama fenómeno, sino la totalidad de los hechos. Wittgenstein entiende por hecho la conexión percibida entre los objetos, que se interrelacionan en un estado de cosas que contiene todas las posibilidades. Para Wittgenstein, el sujeto no existe en el mundo de los objetos y los hechos, porque él mismo "es un límite del mundo" (2009 [1921]: 107). Incluso llegará a afirmar que "el mundo y la vida son una y la misma cosa. Yo soy mi mundo. (El microcosmos)” (2009 [1921]: 105). Este mundo común, en palabras de Garcés (2013), está en vías de desestructuración. 
cual han perdido, poco a poco, su credibilidad. Vidal Castell (2020) expone que no es raro que este periodismo se fundamente en este engaño de estética hiperrealista, porque responde a los intereses de una industria que usa la noción de verdad para reconocerse como altavoz del discurso hegemónico.

El periodismo que bebe de esta simulación global de apariencia realista, como bien ha explicado Vidal Castell (2002), cree que sus narraciones son verdades que reflejan una realidad empírica, y que la periodista es -o aspira a ser- objetiva y puede captar los hechos empíricos tal y como son en la realidad y que a la realidad no le hace falta ninguna otra explicación que no sean los hechos demostrables. Este periodismo renuncia, por lo tanto, a considerar el lenguaje como un aparato intelectual humano que crea mundo en tanto que lo dota de sentido y, por contra, lo considera una mera herramienta automatizada que, si hiciera falta, podría ejecutar un robot, un comunicador no intencional (Martínez Albertos, 1983) que se limita a recoger el hecho y a trasladarlo al lector sin intervenir cognitivamente en el proceso ${ }^{2}$.

Debido a todo ello, y al hecho de que la simulación global que anticipó Baudrillard (1978) ha mutado la última década hacia lo que llamamos hipersimulación (Garde, 2021) -en la que el capitalismo de la vigilancia ha creado, mediante el lenguaje algorítmico, un metarrelato de coherencia planetaria que se encarna en las llamadas plataformas-, el periodismo como disciplina ha sufrido un debilitamiento que ha puesto en duda su tarea empalabradora (Duch, 2009). En definitiva, si en la simulación global, los mass-media servían en bandeja esta narración de la realidad empírica para que el capitalismo la cuantificara, ahora que ya lo está, el metarrelato solo sirve a un interés económico y, por lo tanto, la duplicación fidedigna del real empírico que tan bien ejecutan estos mass-media deja de tener sentido. Así mismo, este negocio capitalista de la verdad (Vidal Castell, 2020) supedita cada vez más el conocimiento de la lengua y habla propias al dominio de un neolenguaje computacional. En las profesiones que viven del empalabramiento del mundo, este neolenguaje computacional llega a tener el poder de una magia, de un conjuro, tal y como recuerda Ed Finn (2018). Así, al final, el periodismo se representa en las redes sociales y en las plataformas como un mero motivo recurrente que ya no interpela.

En este contexto de auge del capitalismo hipersimulado, nos parece pertinente preguntarnos cómo puede el periodismo repensar su rol ${ }^{3}$ en las comunidades y en la construcción de comunidades posibles. Entendemos que cuando las periodistas ejercen su profesión, no pueden captar la realidad tal y como es, sino que captan, como mucho, los fenómenos kantianos, y como poco, el actuar de la materia en un tiempo y un espacio abstractos construidos por convención (Schopenhauer, 2016 [1819]), una hechura (Chillón, 2014) que solo podemos percibir en tanto que intuiciones guiadas por nuestra sensibilidad y nuestro entendimiento. Desde esta perspectiva de raíz subjetivista, la representación -una mera apariencia, una metáfora (Nietszche, 2010 [1896])- no será más que una interpretación, una visión del mundo propio, nunca una copia fiel de la realidad. La representación, por ello,

2 Dicho realismo ingenuo en la lingüística carece de sentido, ya que, como han explicado los pragmáticos, usar el lenguaje es fundamentalmente reconocer y hacer reconocer intenciones (Marín Jorge, 1993: 14). De hecho, la matriz realista ingenua de la epistemología la han puesto en duda incluso realismos fenomenológicos como el de Mario Bunge (1985).

3 Los vínculos entre periodismo y etnografía han aflorado, sobre todo en la academia estadounidense, cada vez que el primero experimenta una crisis epistemológica. Algunos ejemplos son la crisis que vivió el periodismo a finales del siglo XIX y principios del XX, y que origina propuestas como la de los muckrakers. También a la crisis de relato que supone la Gran Depresión de los años treinta en EUA. Ya en los años sesenta del siglo XX se originará el conocido como Nuevo Periodismo. No es objeto de este artículo, pero no podemos dejar de nombrar el impulso del periodismo literario con sensibilidad etnográfica en Iberoamérica a partir de la crisis credibilidad periodística del 2008 y en el que la Fundación Nuevo Periodismo Iberoamericano Gabriel García Márquez -ahora Fundación Gabo- ha tenido un papel fundamental. 
tendrá un carácter mimético (Ricoeur, 1996 [1985]), de semejanza, y necesitará de la intersubjetividad para ser. Dicha visión es necesaria como creadora de sentido en una comunidad.

Entendemos que hasta ahora, en nuestra tradición académica, la reflexión en torno al periodismo literario se ha centrado, sobre todo, en las conexiones con la literatura, específicamente en el uso de los recursos literarios tradicionalmente propios de la novela para construir narraciones facticias (Chillón, 2014). A este debate, queremos sumar un aporte epistemológico desde la etnografía, ya que consideramos que ésta-como metodología y como método ${ }^{4}$ - fortalece las aportaciones del periodismo literario como retrato de nuestro tiempo y espacio, en lo que se refiere a la deconstrucción y construcción crítica de la mirada, gracias al principio de reflexividad.

El llamado periodismo literario -cuyas características son, entre otras, “immersion reporting, accuracy, careful structuring, and a lot of labor, no matter what medium is used" (Sims, 2009: 13)- es el que mejor se acerca a esta perspectiva, como argumentaremos a continuación, y apuntamos que las contribuciones desde la etnografía en torno al concepto de reflexividad aportan al debate claves metodológicas para entender el alcance del periodismo literario.

No podemos considerar el periodismo literario como una escuela de periodismo propiamente dicha o ni siquiera un movimiento. Tampoco podemos encorsetarlo en una sola disciplina. Proponemos entenderlo como una tendencia integrada por un conjunto heterogéneo de obras y autoras que tienen en común cuatro características que consideramos esenciales en nuestra propuesta: a) son miradas a la diversidad social y cultural (Pujadas, et. al, 2010); b) incorporan a sus relatos recursos propios de la literatura; c) las propuestas emergen desde un rechazo abierto a aquello que Albert Chillón describe como "técnicas, rutinas y formas dominantes de los medios convencionales" (2014: 297) y, d) sitúa el trabajo de campo ${ }^{5}$ como pilar fundamental de una propuesta intersubjetiva, que solo es posible desde un sujeto (autora) y en interacción con otros sujetos y para otros sujetos, para construir un mundo común.

Nuestro objetivo no es asimilar etnografía a periodismo literario: son formas de conocimiento diferentes que responden a objetivos distintos. La finalidad de este artículo es enriquecer la reflexión sobre el periodismo literario visibilizando los vínculos con la etnografía y aflorando las aportaciones que la etnografía puede hacer al periodismo literario.

En la era del capitalismo de plataformas (Srnicek, 2018) y de la vigilancia, que debilita el ejercicio del periodismo, consideramos que el debate epistemológico etnográfico en torno a la reflexividad aporta ideas interesantes y nos da pautas para entender cómo se posicionan -o deberían posicionarse- las periodistas en relación a lo que escriben y sobre las personas de las que escriben y cómo empalabran, es decir, cómo convierten su interpretación en discursos públicos a través de narraciones que vehiculan relatos culturales.

4 Mientras que la metodología es una estrategia general, que se compone de métodos concretos, el método debe entenderse como el conjunto de sistemas analíticos que nos permitirán avanzar en nuestro descubrimiento (Rizo, 2010).

5 Es interesante la aproximación que hacer Guber en torno al trabajo de campo, en tanto que decisión subjetiva y continente: “No es un espacio geográfico, un recinto que se autodefine desde sus límites naturales (mar, selva, calles, muros), sino una decisión del investigador que abarca ámbitos y actores; es continente de la materia prima, la información que el investigador transforma en material utilizable para la investigación” (2005: 48) 
La etnografía como metodología de investigación cualitativa se desarrolla a partir de la antropología social británica, la antropología cultural americana y la escuela de sociología de Chicago. Utilizaremos el término etnografía ${ }^{6}$ para referirnos a la metodología cualitativa que desarrolla teorías a través de un trabajo de campo exhaustivo que supone el despliegue de técnicas inmersivas para la descripción de los grupos humanos en su vida cotidiana, es decir, a través de la experiencia directa. La etnografía posibilita participar "en la vida cotidiana de una cultura (cercana o lejana), observar, registrar, mirar de acceder al punto de vista del otro y después escribir aquello que se ha captado" (Augé, 2005: 87). Es decir, permite participar de la vida de las personas, observar qué sucede, escuchar qué se dice y hacer preguntas para recoger vivencias que contribuyan a aportar conocimiento sobre un tema particular (Atkinson y Hammersley, 1994: 15).

En definitiva, en este artículo tratamos de teorizar acerca de lo que aquí acuñamos como mirada reflexiva, a través de la fértil relación entre periodismo literario y etnografía. Dicha noción nos ayuda a replantear no solo la intención, la actitud y la emoción con la que las periodistas se apropian de su oficio, sino que nos permite repensar el periodismo y, por ende, la autoría; e impugnar algunas verdades establecidas por la academia desde postulados positivistas acerca de cómo abordar la tarea de narrar -o representar- el mundo.

\section{Vínculos entre etnografía y periodismo literario: una aproximación comparativa}

Para abordar nuestra propuesta -un ensayo académico teórico sobre las aportaciones de la etnografía al periodismo literario-, se ha llevado a cabo, a través de un ejercicio de prospección bibliográfica, una lectura crítica de conceptos relacionados con el periodismo como representación, mirada, empalabramiento. La propuesta nace de un ejercicio de comparatismo entre aquellas autoras que han vinculado periodismo literario y etnografía.

Para entender los sólidos fundamentos que sustentan los vínculos metodológicos, recuperamos la aportación de Chillón (2014), que bebe tanto del relativismo lingüístico como del realismo fenomenológico. El autor de la Escuela de Bellaterra acuña el concepto palabra facticia para explicar cómo la periodista, cuando ejerce su profesión, no narra hechos empíricos objetivos, tal y como ha defendido -y defiende- una cierta academia periodística de raíz positivista, sino "hechos que son interpretaciones, de cabo a rabo: tramas de sentido que se alimentan de lo evidente, lo comprobable, lo probable y lo plausible [...] entreverados de ficción" (Chillón, 2014: 125).

Según Chillón, no debe entenderse la palabra ficción como un engaño involuntario o deliberada mentira, sino como el "proceder imaginativo sin el que no es posible establecer el sentido de lo que sucede, más allá de sus efectos obvios” (Chillón, 2014: 125). Por ello, entre otros motivos, el autor propone la noción palabra facticia como alternativa a la locución no-ficción, que considera tosca. "Sean científicas o periodísticas, jurídicas o historiográficas, testimoniales o documentales, las mejores expresiones de la facción carecen, en rigor, de esta capacidad de reproducir con objetividad lo sucedido que se les atribuye -con frívola o ingenua inconsciencia, la mayoría de veces-, dado que no pueden ser otra cosa que representaciones, ni más ni menos: mimesis que

6 No es objetivo de este artículo profundizar en las tipologías etnográficas como autoetnografía o etnografía pública y sus vínculos específicos con el periodismo literario. Autores como Gillespie (2012) han abundado en el tema. 
vuelven a hacer virtualmente presente lo ya ocurrido en el pasado, mediante aquella mediación -lingüística, retórica y narrativainherente a cualquiera de los discursos de intención verídica" (Chillón, 2014: 70).

De esta manera, en este artículo entendemos que cuando analizamos un discurso facticio será un discurso verosímil que se habrá escrito con intención verídica, y que será verificable en tanto que es compartido por una comunidad como "hecho institucional" (Chillón, 2014: 123), un entramado de sentido sobre una urdimbre cultural y lingüística que constituye ese mundo común al que se refiere Garcés (2013).

Los vínculos entre el periodismo literario y la etnografía no han pasado desapercibidos ni para la antropología ni para el periodismo. Son muchas las académicas que, desde la teoría tanto antropológica como periodística, han evidenciado los puentes existentes entre ambos (Cramer y McDevitt, 2004; Bird, 2005; Gillespie, 2012; Grindal y Rhodes, 1987; Hermann, 2017; Angulo, 2014; Chillón, 2014). Algunas autoras apuntan a un significativo desdibujamiento entre la etnografía y el periodismo. Elizabeth Bird, por ejemplo, expone que sus objetivos son lo suficientemente parecidos para que una formación en métodos etnográficos pueda ser " a loyal way to broaden the horizons and the richness of journalistic practice" (2005: 307).

Gillespie (2012) expone cuatro aspectos vinculantes entre etnografía y periodismo literario: a) ambos suponen un trabajo inmersivo a largo plazo que debe partir de tener el permiso de los sujetos para observar su vida cotidiana e interaccionar con ellos; b) los textos deben ser veraces y creíbles; c) el foco suele estar en la gente corriente, y d) ambos utilizan recursos literarios para generar historias coherentes para un público. En este último punto, apuntamos que existe una diferencia: el periodismo literario aspira a ser popular, mientras que el de la etnografía tiende a ser académico.

Wolcott (1999) sostiene que, aunque la etnografía no es en sí misma un método claramente definido, un principio central y unificador de todo el trabajo etnográfico es un compromiso con la interpretación cultural. La etnografía supone hacer una inmersión a largo plazo en la vida cotidiana de unas personas con la intención de "describir y comprender la vida social desde la perspectiva de las personas que participan en ella" Wolcott (1999). Periodismo literario y etnografía comparten así el interés por la alteridad, entendiendo ésta como lo hace Krotz (1994), como aquello que nace del contacto cultural y permanente y que pretende captar el fenómeno de lo humano para comprenderlo y convertirlo en palabra.

Si partimos del hecho que ambos tienen su origen en la escritura de viajes ${ }^{7}$, entenderemos también porque etnografía y periodismo literario comparten técnicas de investigación inmersivas de trabajo de campo. Es decir, técnicas como la observación o la entrevista, que permiten reconstruir el significado que el mundo social tiene para las personas y observar, de primera mano, cómo ese significado es aprehendido como movilizador que lleva a cabo las acciones.

De hecho, al visibilizar los puentes, es de justicia recordar que la etnografía juega un papel fundamental en el periodismo de finales de siglo XIX en Estados Unidos ${ }^{8}$ y que, a su vez, no podemos entender el auge de la etnografía en la academia si no traemos a

$7 \quad$ Nos referimos a los libros de viajes que proliferan en Europa a partir del siglo XVI -con especial mención a los libros de mujeres viajeras como Mary Kingsley (1862-1900), Isabella Bird (1831-1904 ), Margaret Fountaine (1862-1940) o Ida Pfeiffer (1797-1858) de los siglos XVIII y XIX- y del periodismo de viajes del siglo XIX, del que Samuel Langhorne Clemens, Mark Twain, (1835-1910) es uno de sus principales representantes.

8 Los muckrakers aparecen en las últimas dos décadas del siglo XIX y la primera del XX como un grupo de periodistas semiorganizado (Filler, 1993) que, recogiendo la herencia de los naturalistas norteamericanos, denuncian la corrupción política y la mala praxis democráticas de ciudad industriales de Estados Unidos, una nación que estaba despertando (Vidal Castell, 2016). En este contexto, los muckrakers investigan la corrupción y denuncian las malas prácticas democráticas de los Estados Unidos en sus artículos. Y lo hacen a contracorriente: cuando la noticia ya es pasado, llevan a cabo un trabajo de investigación de fondo, consultan 
colación que muchos académicos fueron antes periodistas de calle. Robert E. Park, uno de los fundadores de la Escuela de Chicago e impulsor de la sociología urbana, fue periodista ${ }^{9}$ entre 1887 y 1898 antes de dedicarse a la academia. Park comenzó su carrera como reportero de periódicos en Minneapolis, Detroit, Denver, Nueva York y Chicago. Dicha vinculación con el periodismo, que influyó en su trabajo posterior en sociología, se evidencia cuando Park afirma que un sociólogo debe ser "a kind of superreporter, like the men who write for Fortune ... reporting on the long-term trends which record what is actually going on rather than what, on the surface, merely seems to be going on". Así, los sociólogos de Chicago, que registrarían etnográficamente las transformaciones que estaban ocurriendo en las grandes ciudades estadounidenses, o bien practicaron el periodismo o bien tuvieron una estrecha relación con los periodistas de calle (Lindner, 1997).

Así es como, en definitiva, la etnografía recoge, a nuestro juicio, los tres verbos -Ve, vívelo y cuéntalo- con los que Gabriel García Márquez definía el periodismo (Gayà Morlà, 2015). El ve implica ir a buscar los hechos institucionales en la comunidad; el vívelo, desplegar un método inmersivo que, a través de la presencia, y la toma de conciencia de esta presencia, permite pasar de la vivencia a la experiencia para la compresión; y el cuéntalo supone hacer una interpretación cultural -una representación- de lo vivido en aras de crear narraciones comunes que vehiculan relatos compartidos, precisamente para visibilizar aquello que no se ve porque que está oculto o porque es tan común que ha dejado de ser visto.

Unas narraciones que se estructuran en torno a un punto de vista subjetivo, aquello que Geertz resumía con la expresión decir algo de alguna cosa (1973) y que, de nuevo, entendemos como un vínculo entre etnografía y periodismo. Es la presencia en las comunidades y la interacción con los sujetos lo que brinda la posibilidad de pasar de la anécdota a la categoría.

Otro vínculo importante entre uno y otro es aquello que se refiere a lo que en antropología se conoce como descripción densa (Geertz, 1973), es decir, aquella que reconoce las marcas de distinción con las que los sujetos de estudio clasifican su entorno, comprenden y atribuyen sentido del mundo para interpretar estas marcas, al mismo tiempo que genera un discurso sobre aquello que está pasando, sobre la acción humana. En periodismo, Fleta Monzón (2015) recoge el concepto mirada densa, propuesto por Vidal Castell (2008) ${ }^{10}$, "como antídoto a la superficialidad interpretativa, que busca más allá de la apariencia, del estereotipo,

muchas fuentes, verifican la información y la publican con la intención de denunciar aspectos del sistema norteamericano que no funcionan. Lincoln Steffens, uno de las cabezas visibles de la corriente, llegó a decir que para él la pregunta clave ya no era el qué: eran los por qués y el cómo de lo que sucede. Para entender esta corriente debemos entender que estos periodistas llevan a campo un trabajo de campo en los contextos donde han sucedido los hechos institucionales -es decir, regresan a los lugares donde hubo una noticia- y la narración cobra importancia. Los muckrakers surgen en paralelo a la consolidación de la prensa de masas en Estados Unidos -el periodismo se convierte en un negocio industrial y el periodista, en un obrero (Vidal Castell, 2000)- y las revistas necesitan cambiar el modelo de negocio. Collie's, Cosmopolitan, The Masses serán, entre otras, los espacios donde los muckrakers publicarán trabajos como The Shame of the Cities (1904), en que Steffens denuncia la corrupción municipal de St. Louis, Filadelfia, Nueva York, Pittsburgh y Chicago. David Graham Phillips publicó The Treason of Senate en la revista Cosmopolitan, una compilación de reportajes que forzaron al presidente Roosevelt a aprobar leyes como la Pure Food and Drugs Act. Edwin Markham denunció la explotación laboral infantil a Children in Bondage (1914) y Ida Tarbell expuso las formas de negocio especulativas de la Standard Oil Company de Rockefeller a History of the Standard Oil Company (1904). Samuel Hopkins Adams publicó entre 1905 y 1906, en Collier's Weekly, una serie de reportajes sobre la venta de medicamentos peligrosos para la salud con el título The Great American Fraud. Los muckrakers, de hecho, recogen el trabajo iniciado por periodistas como Nellie Bly quien, después de escribir historias para el Pittsburgh Dispatch sobre gente de la calle fue contratada en 1887 por Pulitzer para que trabajara para el The New York World. En el momento en que Bly trabaja para Puiltzer en las paredes del periódico había carteles que anunciaban el cambio en las piezas periodísticas: "The color-the facts-the colour" junto con "Accuracy-accuracy-accuracy". Para Pulitzer, Bly escribirá Diez días en el manicomio, tras hacerse pasar por demente y pasar diez días en un centro psiquiátrico.

9 La página web de la Universidad de Chicago recoge el pasado muckraker de Park: https://www.lib.uchicago.edu/collex/exhibits/university-chicago-centennialcatalogues/university-chicago-faculty-centennial-view/robert-e-park-1864-1944-sociology/

10 La propuesta de Vidal Castell, hecha desde el relativismo lingüístico, se hermana a la noción de mirada reflexiva, que en este artículo teorizamos a partir de las aportaciones de la etnografía. 
del mero dato, para tratar de encontrar elementos profundos del entendimiento humano que permitan un conocimiento más intenso, mejor" (2015: 55).

Un ejemplo reciente de las fronteras borrosas entre ambas disciplinas, que construyen narraciones facticias, es el de la primera mujer periodista en conseguir un premio Nobel de Literatura. En 2016, Svetlana Alexiévich fue premiada, según el propio comunicado de la academia sueca, "por sus escritos polifónicos, un monumento al sufrimiento y la valentía en nuestro tiempo". En el 2016, en plena reflexión sobre la necesidad de hacer un periodismo de calidad en la era digital, David Vidal Castell y Laia Seró Moreno le preguntaban por su método de trabajo. Sin nombrar el enfoque etnográfico, aunque dejándolo traslucir, ella respondía:

\begin{abstract}
Tardo entre siete y diez años a escribir un libro, y converso con 500 o 700 personas. Cuando me encuentro con personas con relatos muy interesantes, entonces las visito cinco o siete veces más. Puede imaginar la cantidad de páginas que tengo [...]. No hago exactamente una entrevista cuando estoy hablando con una persona. Se trata más bien de una charla: empezamos a hablar de la vida, como dos personas que, por casualidad, nos hemos encontrado en la Tierra. Cada uno de nosotros forma parte de una época, de unos acontecimientos, somos testigos de nuestro tiempo, de nuestras esperanzas y utopías. Conversamos sobre todo: sobre cosas serias, pero también sobre la blusa que una lleva o sobre si se ha quemado haciendo un pastel. Es en este momento cuando aparece la esencia del ser humano. Hay que ser una persona muy sencilla y no hay que tener miedo de preguntar cuando no entiendes algo o la desconoces. Tampoco se ha de juzgar, porque todos tenemos cosas que no nos han salido bien a la vida (Vidal Castell y Seró Moreno, 2016).
\end{abstract}

La periodista, como hicieran los llamados nuevos periodistas ${ }^{11}$ en la década de los sesenta y ya habían hecho los muckrakers a caballo del siglo XIX y XX, crea circunstancias (va, escucha, pregunta, experiencia, es decir, las vive) y recurre a los recursos de la literatura para narrar aquello que ha aprehendido: así, la palabra facticia se convierte en la herramienta fundamental para una narración que interpreta el mundo subjetivo de las personas con las que se ha dialogado y empatizado, y de las que se han acumulado muchas vivencias en común, pero es también garantía de haber estado allí, como fuente de verosimilitud y credibilidad para las lectoras. En definitiva, la palabra facticia como un modo de generar conocimiento que visibiliza lo que no es visible, como apuntábamos unas líneas más arriba.

Por todo ello, asumimos que la palabra facticia -para ser verosímil, en definitiva, creíble, es decir, compartida por una comunidad- necesita de un proceso de reflexión de segundo orden -donde la periodista se cuestiona su manera de mirar, su práctica y hasta lo que halla, y hace transparentes sus dudas- sobre lo que implican, para el periodismo, las tres acciones expuestas -ir, vivir y contar-. Es en este punto en el que creemos que debemos reflexionar acerca del principio de reflexividad que proviene de la etnografía, y que trataremos de teorizar en el siguiente apartado.

11 Aunque hay antecedentes notables de los vínculos entre periodismo y literatura anteriores a la década de los sesenta del siglo XX -un ejemplo de ellos es, sin duda, Hiroshima de Hersey-, debemos situar en esa década, y en Estados Unidos, el surgimiento de la corriente conocida como Nuevo Periodismo. Exponemos que, en dicha corriente, los lazos entre periodismo, literatura y etnografía cobran especial sentido. Las obras de Tom Wolfe o Gay Talese, entre otros, suponen ejemplos maravillosos de un periodismo que se sumergía allí donde pasaban las cosas (Wolfe, 1973) y que hizo de la descripción y el diálogo cotidiano dos de sus armas más poderosas. También Norman Mailer, Joe McGinnis, Barbara L. Goldsmith... escribieron sobre lo que vieron con sensibilidad etnográfica y los recursos narrativos propios de las corrientes naturalistas de finales del siglo XIX. Los espacios que abrazaron con mayor profusión los textos del Nuevo Periodismo fueron las revistas y los magazines especializados en asuntos sociales y culturales: Esquire, New York, Ramparts, The New Yorker, Playboy, Harper s Magazine, y otras nacidas en el seno de la contracultura de aquellos años, como Rolling Stone y The Village Voice. Es de obligada mención, sin embargo, que la presencia del periodismo literario de los sesenta se manifestó también en forma de libros firmados por los mismos reporteros, como Gay Talese, Terry Southern, Hunter S. Thompson, y que, a pesar del choque que propugnaban contra las formas de hacer del periodismo tradicional, tal fue el impacto de los nuevos periodistas que los grandes diarios también alojaron algunos de sus artículos. 


\subsection{Aportaciones al periodismo literario: la mirada reflexiva, a partir del principio de reflexividad etnográfica}

Antes de profundizar en la noción de reflexividad y en cómo puede ayudar a las periodistas a situar su praxis, nos parece pertinente recoger 13 puntos a los que alude la periodista Leila Guerriero (2010) para describir los pilares sobre los que se fundamenta el periodismo narrativo que, en este texto, consideramos sinónimo del periodismo literario:

1. Toma recursos de la ficción para contar una historia real y monta una arquitectura tan atractiva como la de una buena novela.

2. Se construye sobre el arte de mirar.

3. Es lo opuesto a la objetividad. Es una mirada, una visión del mundo, una subjetividad honesta. Toda pieza de periodismo es una edición de la realidad.

4. Para ver no solo hay que estar: para ver, hay que volverse invisible.

5. Solo permaneciendo se conoce, y solo conociendo se comprende, y solo comprendiendo se empieza a ver. Y solo cuando se empieza a ver, se puede contar.

6. En esta nueva forma de periodismo la unidad de trabajo no es ya el dato, sino la escena.

7. Hay que tener algo para decir.

8. Hay que conocer la realidad que se va a narrar. Saber de qué se habla.

9. Hay que descubrir cuál es la mejor forma de contar la historia.

10. Es instrumento para pensar, crear, ayudar. El periodista escribe para producir un efecto.

11. Escribir sobre gente común, en circunstancias absolutamente extraordinarias y gente extraordinaria en circunstancias comunes.

12. La clave del periodismo narrativo reside en que, hablándonos de otros, nos habla de nosotros mismos.

13. De todos los recursos de la ficción que el periodismo puede usar, hay uno que le está vedado: el recurso de inventar.

Los puntos 2, 3, 4, 5, 6, 7, 8, 10 y 12 nos conducen a las siguientes preguntas: ¿Qué significa el arte de mirar? ¿Cómo se acerca el periodismo literario al mundo subjetivo de las personas y las comunidades a las que se acerca? ¿Qué implica este acercamiento? ¿Cómo los mundos subjetivos de estas personas pueden contribuir, mediante las narraciones periodísticas, a construir un mundo común? ¿Qué rol se arroga la periodista y cómo lo ejerce? Son todas ellas preguntas que las periodistas literarias han respondido, a veces desde la intuición, a veces desde la práctica del oficio (Kapuscinsky, 2003; Terzani 2006; Guerriero, 2010; Salcedo Ramos, 2011). Por ello, creemos, puede resultar necesario recuperar la tradición académica en torno a la reflexividad etnográfica que, consideramos, es una base fundamental para el periodismo literario, que hace del trabajo de campo -la experiencia de la presencia- su principal motor de acción reflexiva.

Hasta tal punto la reflexividad etnográfica es útil al periodismo literario que Cramer y McDevitt (2004) proponen poner en práctica un "periodismo etnográfico". Pese a que no nos parece necesario asumir la nomenclatura de estos autores, ya que entendemos que todo periodismo literario parte de una sensibilidad etnográfica, sí recogemos lo que entienden por reflexividad aplicada al periodismo literario: "This reflexivity requires that reporters become self-conscious about their social locations in relation to the individuals and groups they write about. Autonomous reporters would realize that to pursue ethnographic journalism, they must in some ways transcend not only professional conventions and reporting habits also their own demographic profiles" (2004: 131). 
En este sentido, el principio de reflexividad aporta una consciencia cada vez más clara del riesgo que supone objetivizar a las personas y las culturas, además de imponer el mundo subjetivo de la autora en las experiencias de las otras. Debemos remarcar, pues, que el principio de reflexividad supone replantearse la forma y el modo de producir conocimiento, tomando distancia de posiciones positivistas. De este modo, el punto de partida de la reflexividad implica considerar la existencia de un mundo común narrado por hechos institucionales; implica interaccionar, observar y participar intersubjetivamente con una comunidad en determinadas circunstancias espacio-temporales y, desde allí, considerar a la periodista como parte de ese mismo mundo que se estudia (Amegeiras, 2006: 115) mientras se está presente y entender que ésta empalabra desde donde se sitúa para visibilizar la humanidad que compartimos. Lo expresaba de esta manera Restrepo (2012): "Hay un elemento en común [entre la periodista y las personas para las que escribe]: la humanidad. Si pierdes la humanidad de ese público, estás dando golpes en el vacío. Tienes que hablarle a un público que está reunido por su condición humana, por tanto, tienes que humanizar tu contenido”.

Entendemos que, para avanzar en la reflexión, antes debemos acercarnos a la noción de mirada -uno de los sustratos que nutren el periodismo literario (Angulo, 2014; Chillón, 2014; Vidal Castell, 2020)- para vincularla al concepto de reflexividad y desarrollar lo que aquí acuñamos como mirada reflexiva. Desde la práctica periodística la mirada se ha definido más como la intención de la autora que como una metodología. Sin embargo, la mayoría de autores del llamado periodismo literario sí desarrollan una aproximación más compleja desde diferentes ángulos. Angulo, por ejemplo, aborda la cuestión del mirar y de la mirada de las cronistas -y que en este artículo consideraremos periodistas literarias- más allá de la simple intención y escribe:

Los cronistas utilizan la mirada con más intensidad que la pluma o las teclas del ordenador. Saber qué mirar. Saber cómo mirar. Pero decir "mirar" no es decir mucho, porque "mirar" no es ver, es pensar. Es centrar, focalizar, encuadrar. Mirar también es escuchar, que no oír. Poner una voz en off para hacer oír la de los verdaderos protagonistas. Mirar es atender a los lados sin perder de vista el frente. Prever el futuro y echar un vistazo atrás de vez en cuando. Mirar es documentarse y reportar, adentrándose en las vidas ajenas a través de zoom in y realizar panorámicas desde la distancia mediante zoom out. [...] Mirar no es despreciar los tiempos: pasado, presente y futuro. Mirar es traducir. Es percibir los espacios, atender al ángulo muerto, al fuera de campo, a lo liminal, a la fisura. Mirar es contar con estas variables espacio-temporales, cuando parece que la ceguera cotidiana se ha generalizado por saturación informativa (Angulo, 2013: 7).

Así, debemos precisar que la mirada es inherente a la construcción del mundo narrado y se construye "a partir de métodos de observación, descripción y análisis; utiliza instrumentos técnicos y conceptuales que configuran y reconfiguran una forma de ver el mundo. La imagen es el producto de una mirada sobre el mundo. La investigación etnográfica se sirve generalmente de la palabra para representar la realidad social y la experiencia del etnógrafo" (Ardèvol, 1994:8). En el mismo sentido, Rosana Guber (2005) se refiere al proceso etnográfico como ese "abrir la mirada, es decir, la sensibilidad perceptiva, la capacidad de sorpresa y de perplejidad 'y también' abrir los sentidos, relativizando certezas y dando entrada a nuevas definiciones y perspectivas”. Así, expone:

El trabajo de campo implica un pasaje de la reflexividad general, válida para todos los individuos en tanto seres sociales, hacia la reflexividad de aquellos que toman parte en la situación de trabajo desde su rol de investigador. Pero este pasaje no es meramente secuencial, es decir que el investigador no dispone y conoce primero su propia reflexividad y después accede a la de los informantes. Su propia reflexividad, al contrastarse con la de los sujetos que estudia, se resignifica y encuentra un nuevo lugar. A los efectos del grado de conocimiento, es muy probable que el investigador sepa más de su reflexividad después de haberla contrastado con la de sus informantes que antes del trabajo de campo (Guber, 2005: 50). 
En definitiva, ser consciente de que la mirada es un filtro que permea tanto la concepción de una obra periodística como la interpretación que se hace de la experiencia permite constatar que la periodista literaria entiende que el lugar desde el que mira para empalabrar es moldeado por un proceso histórico-cultural (Haraway, 1998 y Harstock, 1983)12 que la sitúa. La reflexividad nos ayuda también ser conscientes de los privilegios y de los diferentes sistemas de dominación de los que participa quien empalabra.

Por ello, debemos comprender la reflexividad como un proceso de reflexión de segundo orden (Ibáñez, 1994) que se produce "cuando un sistema observador toma distancia para observarse a sí mismo y, en un distinto nivel de observación observa los procesos y relaciones del sistema” (Aguado, 2003: 279). Como ya hemos apuntado, dicho proceso, por un lado, implica repensar los imaginarios sociales, los privilegios y los ejes de opresión desde los que construye su mirada. Por el otro, ser consciente del propio conocimiento situado permite desarrollar una mirada más allá de la experiencialidad e incorporar la interseccionalidad (Davis, 2008) necesaria para empalabrar sin ideas preconcebidas, prejuicios o estereotipos.

La observación dialéctica de la alteridad hecha desde una mirada reflexiva también supone reconocer la noción de comunidad. Aquello que Malinowski definía como comprender las cosas desde el punto de vista del nativo y que Geertz puntualiza como: desde el punto de vista del nativo de una cultura sin convertirse en nativo (Geertz, 1995). En este sentido, desde nuestro punto de vista, la mirada reflexiva supone un ejercicio participativo en el que los sujetos, mediante un ejercicio de intersubjetividad ${ }^{13}$, intentan interpretar, narrar y crear un mundo común. El ejercicio es dialógico y es fruto de una escucha activa y de la asunción de que la periodista no es ni pasiva ni externa a aquello que vive y narra.

En esta propuesta, el principio de reflexividad emerge como un punto de partida y, a la vez, como una actitud que debemos tener para enfrentarnos a la tarea de interpretar, y que nos permite, luego, empalabrar. Así, la mirada como proceso reflexivo podemos teorizarla en tres etapas: tiene que ver con un proceso de toma de conciencia de la periodista que se acerca al campo y se relaciona con las personas que habitan un espacio en un tiempo determinado, así como al método que escoge para observar y participar del campo y también de la inventio. Detengámonos en este último punto: la mirada reflexiva es, pues, determinante en el planteamiento o la inventio y, por tanto, en la confección previa a la escritura. La autora debe ser consciente constantemente que esta mirada representa un filtro que determinará la interpretación y la comprensión que haga de los hechos desde el minuto cero. Es decir, en la lectura que haga de las narraciones que ha recogido, en la selección de los elementos que considere más oportunos visibilizar e, incluso, en la elección de los procedimientos de investigación.

A través de una mirada reflexiva, la práctica experiencial que supone el trabajo de campo se convierte en una descripción densa (Geertz, 1973): la mirada reflexiva permite reconocer las marcas de distinción con las que las personas que pertenecen a una comunidad -a un espacio en un tiempo determinado- clasifican su entorno, comprenden y atribuyen sentido del mundo para interpretar estas marcas, al mismo tiempo que genera un discurso sobre aquello que está pasando, sobre la acción humana, y ellas mismas. En otras palabras: la mirada reflexiva, a través de una descripción densa, otorga la capacidad de comprender cómo

12 No es objeto de este texto desarrollar el pensamiento feminista de Haraway (1998) y Harstock (1983). Aun así, hacemos un apunte a pie de página para exponer que ambas autoras seleccionan la raza, el género y la clase para poner la mirada en los colectivos que, desde posiciones patriarcales, privilegiadas y eurocéntricas se invisibilizan.

13 El debate, en el ámbito etnográfico, surge a partir de la publicación de los diarios personales de Malinowski, a finales de los sesenta. 
han aprehendido la doxa ${ }^{14}$ los sujetos para, precisamente, a través de la narración periodística, generar dudas, sospechas, puntos de fuga en torno a ésta. Y dicha narración, más que reproducir un relato cultural hegemónico, se convierte en sí mismo en una narración cultural que contrarresta la doxa de dicho relato hegemónico.

La propuesta en torno a la mirada reflexiva revela la urgencia de que la autora se asuma como una intelectual empalabradora. De esta manera, Casamajó entiende a la autora de periodismo literario como un "ventrílocuo" que puede impostar varias voces a la vez: "La de l'autor empíric-l'individu que escriu-, que és una instància real però lingüísticament virtual; la de l'autor implícit -versió de l'autor empíric inferida pel lector a partir del relat-, que és una instància virtual efectivament i lingüísticament; $i$ la del narrador -la veu que condueix el relat-, que és una instància fictícia però lingüísticament real" (2002: 143).

Entender este ejercicio de ventriloquía supone reconocerse como sujeto intelectual, cuya subjetividad "ya no debe esconderse". "Debe aflorar como parte de la metodología y buscar las fronteras de la praxis periodística en la ética profesional" (Gayà Morlà, 2015: 154). Dicha ética periodística nos permite entender, por tanto, el periodismo como "a link to human flourishing, commitment to the common good, reporting as the defining activity ofjournalism, a desire to make a difference, and a way to make a living" (Borden, 2007: 49-50).

\section{A modo de conclusión: retos y debates}

Un periodismo basado en una mirada reflexiva es capaz de argumentar una metodología y un método, y construye, por ello, una alternativa al metarrelato del capitalismo acelerado de la era digital. Lo expuesto nos regresa a una temporalidad que podemos habitar de manera coherentemente humana, es decir, entendiendo que el contexto y la interacción intersubjetiva son los trazos de coherencia de una comunidad. Entendemos que la propuesta presentada cambia radicalmente las lógicas de la hipersimulación: permite a la comunidad apropiarse del aquí y ahora y abandonar el presente continuo que no nos interpela y, además, otorga credibilidad al periodismo. Es función del periodismo literario poner en evidencia el espejismo que construye el capitalismo en las plataformas. La mirada reflexiva nos da el marco epistemológico para hacerlo.

Aun así, entendemos que algunos aportes en torno a la mirada reflexiva expuestos anteriormente son una afrenta a la definición canónica positivista del periodismo y también pueden suponer dilemas éticos para las periodistas literarias que asuman la mirada reflexiva como principio de acción e interpretación, más allá de un subjetivismo ontológico.

14 Para acercarnos al concepto de doxa recuperamos la teoría de los campos de Pierre Bourdieu. El autor define el campo como un espacio relativamente autónomo, con objetivos propios, con jugadores que compiten entre sí (a veces ferozmente) y que siguen estrategias diferentes según estén posicionados y su capacidad de apuesta (capital) y que, al mismo tiempo, están interesados en jugar y reconocen que vale la pena jugar. "Un campo no es simplemente una estructura muerta o un sistema de 'lugares vacíos' [...], sino un espacio de juego que solo existe en cuanto tal, en la medida en que existan también jugadores que entren en él, que crean en las recompensas que ofrece y que las busquen activamente" (Bourdieu y Wacquant (2008) [1992]: 26). El habitus es "aquel sistema de disposiciones durables, transferibles estructuradas, predispuestas a funcionar como estructuras estructurantes; esto es, como principios de generación y de estructuración de prácticas, así como de representaciones que pueden ser objetivamente reglamentadas y regulares, sin ser en absoluto el producto de la obediencia a las reglas; y que se adaptada objetivamente a su meta sin presuponer la visión consciente de los fines y de destreza explícita de las operaciones necesarias para lograrlas; siendo todo aquello colectivamente orquestado, sin ser el producto de la acción organizadora de un director de orquesta" (Bourdieu, 1991 [1980]: 92). Es esclarecedora la aportación que hace González al concepto de doxa cuando asegura que ésta alude "a los esquemas de interpretación de primer orden, generalmente irreflexivos, aprendidos y vividos como naturales, obvios y evidentes. Operan en la base de toda práctica y se aprenden o asumen por simple familiarización" (1998: 161). 
Asumir que el periodismo es una actividad simbólica que vehicula relatos a través de interpretaciones supone poner en duda convenciones e ideales sobre los que se ha fundamentado la teoría periodística: nos referimos a la verdad demostrable como justificación de la práctica y del discurso, la supuesta objetividad o la neutralidad de las periodistas y la búsqueda de hechos reales en un presente continuo, que resulta ser una ficción. Asumir la mirada reflexiva es, sin duda, alejarse del fetichismo del presente y deconstruir prácticas derivadas como son la inmediatez (Deuze, 2005), la rapidez, la urgencia, la búsqueda de los hechos empíricos.

La propuesta supone entender que: a) la periodista es una intelectual que hace una tarea interpretativa; b) el periodismo se ocupa de poner en común y de dotar de sentido un mundo compartido y regresa en presencia a lo social; y c) se recupera la dimensión creativa y cultural del relato como una interpretación subjetiva y posible.

La tarea periodística es un proceso en el que se pone de manifiesto que la mirada de la autora es reflexiva y que ésta se construye a partir de un proceso de reflexión de segundo orden y en relación con los informantes, que ahora son personas con las que se relaciona de manera intersubjetiva. Un tema que no es baladí, sobre todo si tenemos en cuenta el arraigo positivista que destilan las aulas de periodismo. Nuestra propuesta pone de relieve que el periodismo es una disciplina creativa de interpretación y configuración de sentido.

Así pues, uno de los retos es redefinir el rol de las periodistas en lo que se refiere a la distancia entre éstas y las personas sobre las que se escribe, así como aquello que entendemos por autoría. Proponemos que la autora no solo lo sea por el discurso que propone, sino por asumir la responsabilidad como periodista en la tarea de dotar de sentido y de construir un mundo común. Queda aún por reflexionar y discutir aquello que en arte se denomina autoría colectiva y de qué manera pueden participar las personas sobre las que se escribe en el discurso final.

En el trabajo de campo, y en el ejercicio de empalabramiento, la fuente deja de ser fuente para convertirse, como ya se ha apuntado, en persona, y ésta se muestra y habla desde su intersubjetividad individual y grupal. La periodista, en cambio, deja de justificar su trabajo como el de una observadora externa para asumir que su actividad es subjetiva y que su presencia (e interacción con la comunidad) afecta a las personas de las que pretende escribir. De ellas, ya no se extrae información, sino que se busca -en actitud de aprendizaje- qué sentido dan a su mundo (Cramer y McDevitt, 2004) para, a través de un discurso que nace para ser público, abordar la diversidad de sentidos y significaciones posibles, más allá del metarrelato que difunde el discurso hegemónico en una comunidad.

La separación entre periodista y fuentes deviene una impostura, un artificio sin sentido. La mirada reflexiva implica una relación cercana, incluso empática, con las personas sobre las que se construirá un discurso público. Dicha reflexión, de nuevo, es un reto, ya que esta forma de relación entre periodista y personas entra en contradicción con uno de los principios del periodismo: aquel que sitúa la responsabilidad de las periodistas con las lectoras y con el interés público, más que con los sujetos sobre los que se escribe. A la periodista no le queda otro remedio que ser honesta con las comunidades a las que se acerca, siendo la honestidad un tema capital en los estudios de periodismo, porque sin una actitud honesta se genera una situación de traición (Malcolm, 2012) con aquellas personas con las que ha interaccionado.

La propuesta también expande aquello que se configura como interés periodístico: el trabajo de campo convierte lo cotidiano en narración, más allá del conflicto. Entendemos que más que crear un acontecimiento basado en el conflicto, la mirada reflexiva 
permite a la periodista identificar estructuras de significación y explicar y poner en duda la cultura, y los mecanismos de poder que la configuran, así como proponer posibles formas de emancipación.

El trabajo de campo en el periodismo literario requiere también de una presencia más prolongada. Recordemos el punto de 5 de Leila Guerriero: "Solo permaneciendo se conoce, y solo conociendo se comprende, y solo comprendiendo se empieza a ver. Y solo cuando se empieza a ver, se puede contar".

El discurso periodístico, así, no se configura sobre la base de una narración dramática y espectacular de unos hechos empíricos estructurados con una doble intención: entretener e informar. Emerge como una posibilidad de comprensión y es configurado por una suma de detalles (no de anécdotas) interpretados según el punto de vista construido por la autora.

Mirar presupone un modo de ver, tal y como diría John Berger (2013[1972]), que equivale al proceso complejo de abordar ya no la realidad, sino la interpretación que se ha hecho en y de un espacio y un tiempo que la periodista ha compartido con unas personas, y que convertirá en un discurso público. Es decir, un discurso que implica empalabrar lo aprendido en dicho espacio y tiempo con el objetivo de crear -como diría Gabriel García Márquez- “un retrato crítico de nuestro tiempo", en aras de construir comunidad.

\section{Agradecimientos}

Artículo traducido al inglés por Richard Thompson.

\section{Referencias bibliográficas}

Aguado, J. M. (2003). Comunicación y cognición: bases epistemológicas de la complejidad. Comunicación Social Ediciones y Publicaciones.

Angulo, M. (Coord) (2014). Crónica y Mirada. Aproximaciones al periodismo narrativo. Libros del K.O.

Ardèvol, E. (1994). La mirada antropológica o la antropología de la mirada: De la representación audiovisual de las culturas a la investigación etnográfica con una cámara de vídeo. [Tesis Doctoral]. Universitat Autònoma de Barcelona.

Augé, M. (2005). La mère d'Arthur. Fayard.

Atkinson, P. y Hammersley, M. (1994). Ethnography and participant observation. En N. K. Denzin y Y. Lincoln (Eds.), Handbook of qualitative research (pp. 248-261). CA: Sage.

Baudrillard, J. (1978). Cultura y Simulacro. Editorial Kairós.

Berger, J. (2013). [1972]. Modos de ver. Gustavo Gili.

Bird, E. S. (2005). The journalist as ethnographer? How anthropology can enrich journalistic practice. En E. Rothebuhler y M. Coman (Eds.), Media Anthropology, (pp. 301-308). Sage.

Borden, S. L. (2007). Journalism as practice. Macintyre, virtue ethics and the press. Ashgate.

Bourdieu, P. (1991) [1980]. El sentido práctico. Taurus.

Bourdieu, P. y Wacquant, L. (2008) [1992]. Una invitación a la sociología reflexiva. Siglo XXI. 
Bunge, M. (1985). Realismo y Relativismo. Alianza Editorial.

Casamajó, G. (2002). Les veus del ventríloc. Proposta de fonamentació teòrica i metodològica per a l'estudi de la presencia de l'autor en el relat periodístic escrit. [Tesis de Maestría]. Universitat Autònoma de Barcelona.

Chillón, A. (2014). La Palabra Facticia. Literatura, Periodismo y Comunicación. Aldea Global.

Cramer, J. y McDevitt, M. (2004). Ethnographic Journalism. En H. Iorio, S. (Ed.), Qualitative research in Journalism. Taking it to the streets (pp. 127-145). Lawrence Erlbaum Associates.

Davis, K. (2008). Intersectionality as buzzword : A sociology of science perspective on what makes a feminist theory successful. Feminist Theory, 9 (1), 67-85. http://doi.org/ 10.1177/1464700108086364

Deuze, M. (2005). What is journalism? Professional identity and Ideology of Journalists Reconsidered. Journalism Theory Practice and Criticism, 6 (4), 443-465.

Duch, Ll. (2009). Mito, Interpretación y Cultura. Herder.

Duch, Ll. (2019). Vida Cotidiana y Velocidad. Herder.

Filler, L. (1993). The Muckrakers. Stanford University Press.

Finn, E. (2018). La Búsqueda del Algoritmo. Imaginación en la Era de la Informática. Alpha Decay.

Fleta Monzón, D. (2015). Periodismo Mágico. Propuesta de descripción de los recursos compositivos y estilísticos de la crónica deportiva escrita desde la perspectiva de los estudios literarios aplicados al realismo mágico [Tesis de Doctorado]. Universitat Autònoma de Barcelona. http://hdl.handle.net/10803/291822

Garcés, M. (2013). Un mundo común. Ediciones Bellaterra.

Garde, C. (2 de julio de 2021). Atrapats dins la gàbia de miralls: la comunicació de xarxes en temps de Covid-19. Una proposta de glossari per revertir les inèrcies del capitalisme de la vigilància [Comunicación]. IV Congrés Internacional de Recerca en Comunicació, Universitat Autònoma de Barcelona.

Gayà Morlà, C. (2015). El Periodismo de Interacción Social, una propuesta de dinamización del campo periodístico. La aplicación en el caso de las tripulaciones abandonadas en el Mediterráneo. [Tesis de Doctorado]. Universitat Autònoma de Barcelona.

Geertz, C. (1973). Thick description: Toward an interpretive theory of culture. En The interpretation of cultures: selected essays (pp. 3-30). Basic Books.

Geertz, C. (1995). La interpretación de las culturas. Gedisa.

González, J. (1998). Educación, tecnología y cultura: una propuesta de investigación exploratoria. Estudios sobre las Culturas Contemporáneas. Época II, IV, (7), 153-164.

Gillespie, B. (Otoño 2012). Building Bridges between Literary Journalism and Alternative Ethnographic Forms: Opportunities and Challenges. Literary Journalism Studies, 4(2), 67-79.

Grindal, B. y Rhodes, R. (1987). Journalism and anthropology share several similarities. Journalism Educator, 41(4), 11-13.

Guber, R. (2005). La etnografia: método, campo y reflexividad. Norma. 
Guerriero, L. (2010). ‘¿Qué es el periodismo literario? [Conferencia]. Narrativa y periodismo. Fundación Santillana, la Fundación Universidad Internacional Menéndez Pelayo y el Instituto Tecnológico de Monterrey.

Haraway, D. (1998). Situated Knowledge: The Science question in feminism and the privilege of a partial perspective. Feminist Studies, 14, (3), 575-599.

Hartsock, N.C.M. (1983). “The Feminist Standpoint: Developing the Ground for a Specifically Feminist Historical Materialism”. En S. Harding y M.B. Hintikka (Eds.), Discovering Reality: Feminist Perspec- tives on Epistemology, Metaphysics, Methodology, and Philosophy of Science (pp. 283-310), Synthese Library. Springer Netherlands, Dordrecht. https://doi.org/10.1007/0-306-48017-4_15 Heidegger, M. (1986) [1927]. El Ser y el Tiempo. Fondo de Cultura Económica.

Hermann, A. K. (2017). J-schoolethnography. Journalism Studies. 18:2, 228-246. https://doi.org/10.1080/1461670X.2015.1043322 Ibáñez, J. (1994). El regreso del sujeto: la investigación social de segundo orden. Siglo XXI Editores.

Jameson, F. (1991). El Posmodernismo o la Lógica Cultural del Capitalismo Avanzado. Paidós.

Kapucinsky, R. (2003). Los cinco sentidos del periodista. Fondo de Cultura Económica.

Krotz, E. (1994). Alteridad y pregunta antropológica. Alteridades, 4(8),5-11. ISSN: 0188-7017.

Kant, E. (2007). [1781]. Crítica de la Razón Pura. Editorial Losada.

Lindner, R. (1997). The Reportage of urban culture: Robert Park and the Chicago School. Cambridge University Press.

Malcolm, J. (2012). El periodista y el asesino. Gedisa.

Marín Jorge, M. (1993). El Discurso de la Información. En: Grupo Andaluz de Pragmática (Ed.), Estudios Pragmáticos: Lenguaje y Medios de Comunicación (pp. 11-27). D. L.

Martínez Albertos, J. L. (1983). Curso General de Redacción Periodística. Mitre.

Nietzsche, F. (2010) [1896]. Sobre Verdad y Mentida en Sentido Extramoral y otros Fragmentos de Filosofía del Conocimiento. Tecnos. Pujadas, J. J; Comas, D.; Roca, J. (2010). Etnografía. UOC.

O’Reilly, K. (2009). Key Concepts in Ethnography. CA: Sage Publications Inc.

Restrepo, J. D (2012). Hay un elemento en común entre los internautas, su humanidad. Comunicació i Responsabilitat Social. Institut de la Comunicació. https://comress.org/2012/05/20/javier-dario-restrepo-hay-un-elemento-comun-entre-los-internautas-su-humanidad/

Ricoeur, P. (1996) [1985]. Tiempo y Narración III. El Tiempo Narrado. Siglo XXI.

Rizo García, M. (2010). Intersubjetividad y diálogo intercultural. La sociología fenomenológica y sus aportes a la comunicación intercultural. Comunicación y medios, (21), 13-23. https://doi.org/10.5354/0719-1529.2011.17446

Salcedo Ramos, A. (2011). Quince reflexiones de paeriodismo narrativo para compartir con los estudiantes. Taller de Periodismo Cultural. FNPI. http://bicentenario.fnpi.org/meteriales/quince_reflexiones_sobre_periodismo_narrativo_para_compartir_con_ los_estudiantes.pdf

Schopenhauer, A. (2016) [1819]. El Mundo como Voluntad y Representación I. Editorial Trotta. 
Sims, N. (Otoño, 2009). The Problem and the Promise of Literary Journalism Studies. Literary Journalism Studies 1, no. 1, 7-16.

Srnicek, N. (2018). Capitalismo de Plataformas. Caja Negra Editora.

Terzani, T. (2006). El fin es mi principio. Un padre, un hijo y el gran viaje de la vida. Maeva Ediciones.

Vidal Castell, D. (2002). La transformació de la teoria del periodisme: una crisi de paradigma? Anàlisi, (28), 21-54. https://ddd. uab.cat/pub/analisi/02112175n28/02112175n28p21.pdf

Vidal Castell, D (2008). La “mirada densa”: el tránsito del caos al cosmos en la narración periodística. En: La periodística como disciplina universitaria. (pp. 171-178). Sociedad Española de Periodística.

Vidal Castell, D. (2016). Theodore $i$ la colla dels aplega-merdes. SomAtents. http://somatents.com/somaccio/ el-vell-theodore-i-la-colla-dels-aplega-merda/

Vidal Castell, D. (2020). Las Fake-news como Recurso de Legitimación de los Medios Convencionales. Análisis Discursivo de Artículos Publicados en los Principales Diarios de Barcelona (2017-2019). A: VV.AA. Actas del VII Congreso Internacional de la AE-IC. Comunicación y Diversidad (pp. 2591-2615). Asociación Española de Investigación de la Comunicación.

Vidal Castell, D., y Seró, L. et al (2016). Totes les veus d'Svetlana. SomAtents, http://somatents.com/magazine/ totes-les-veus-de-svetlana/.

Wolcott, H. F. (1999). Ethnography: A Way of Seeing. CA: AltaMira Press.

Wolfe, T. (2012) [1973]. El nuevo periodismo. Anagrama.

Wittgenstein (2009 [1921]). Tractatus lógico-Philosophicus. Investigaciones filosóficas sobre la certeza. Gredos.

Zuboff, S. (2020). La Era del Capitalismo de la Vigilancia. Paidós. 
